\title{
Intra-arterial Therapy for Acute Ischemic Stroke
}

\author{
Alex Abou-Chebl
}

Published online: 30 June 2011

(C) The American Society for Experimental NeuroTherapeutics, Inc. 2011

\begin{abstract}
Intra-arterial therapy (IAT) for acute ischemic stroke refers to endovascular catheter-based approaches to achieve recanalization using mechanical clot disruption, locally injected thrombolytic agents or both. IAT may be used in addition to intravenous tissue plasminogen activator (tPA) or in patients who do not qualify for tPA, usually because they are outside the approved 3-h timeframe window or have contraindications, such as elevated international normalized ratio or partial thromboplastin time. Recanalization rates correlate with clinical improvement, and with the newest catheters it is possible to achieve recanalization in roughly $80 \%$ of patients treated. However, while the catheters are approved by the Food and Drug Administration, there are still no randomized trial data demonstrating the role of current IAT therapy vs either tPA or standard management. IAT is reserved for patients with large artery occlusions in the basilar, distal carotid, or proximal middle cerebral arteries. Imaging the penumbra using magnetic resonance imaging or computed tomographic perfusion is currently the most frequently used way to identify patients who might benefit. However, the imaging and clinical criteria for identifying which patients benefit, and perhaps more importantly those who will do poorly despite IAT, remain unclear.
\end{abstract}

Keywords Endovascular. Catheter- Intra-arterial · Mechanical clot disruption · Intervention · Thrombolysis . Recanalization $\cdot$ Reperfusion $\cdot$ MERCI $\cdot$ Penumbra

Electronic supplementary material The online version of this article (doi:10.1007/s13311-011-0059-8) contains supplementary material, which is available to authorized users.

A. Abou-Chebl $(\square)$

Department of Neurology,

University of Louisville School of Medicine,

Louisville, KY 40202, USA

e-mail: a0abou03@louisville.edu

\section{Introduction}

Acute ischemic stroke (AIS) is the leading cause of adult disability and the third leading cause of death in the United States, yet until recently it was considered to be untreatable. Major advances in endovascular therapy in the past 10 to 15 years have now made treatment a reality with the potential for remarkable recovery. Although intravenous (IV) thrombolysis with tissue plasminogen activator (tPA) has been approved by the Food and Drug Administration (FDA) since 1996, it has been used in only 3 to $4 \%$ of ischemic stroke patients due to major limitations and a narrow 3- to 4.5-h treatment window [1, 2]. The benefit of IV tPA is limited, both in regard to clinical and recanalization efficacy, particularly for large vessel occlusions, the most common cause of severe and fatal AIS [3, 4]. Large vessel occlusions are however amenable to endovascular therapy, not unlike acute coronary syndromes presenting as ST segment elevation myocardial infarction, which are better treated with percutaneous interventions rather than IV thrombolysis [5]. However, unlike acute coronary artery syndromes, which are most often caused by an atherosclerotic plaque rupture, AIS and transient ischemic attack causes are more varied and include cardiac embolism, cervical atherosclerosis with thrombosis or artery-to-artery embolism, intracranial atherosclerosis leading to thrombosis or hypoperfusion, perforator occlusion due to lipohyalinosis, arteriosclerosis, or embolism, and spontaneous or traumatic arterial dissection, vasculitis, or venous thrombosis, among others. As a consequence of this heterogeneity, no single specific therapy will be effective for all cases especially when the numerous factors (as follows) affecting neurological outcome are considered. These factors determine the risk of intracerebral hemorrhage (ICH), the most feared and devastating complication associated with all treatments for ischemic stroke [6-8]. The risk of ICH is the single greatest limiting factor for AIS recanalization therapy because there is no effective treatment for $\mathrm{ICH}$, and when it 
complicates AIS revascularization treatment, mortality is high. Stroke specialists, therefore, need to have a thorough understanding of all clinical, anatomical, pathological, and radiological aspects of AIS and the spectrum of $\mathrm{ICH}$ to make reasonable decisions regarding intra-arterial therapy (IAT) in every patient.

\section{Indications}

The indications for endovascular treatment, also known as IAT, for AIS are evolving. Because there is no FDA-approved IAT for stroke, there is a great deal of variability between experts in the field regarding the indications. In general, any patient with AIS presenting in $<3 \mathrm{~h}$ should be offered IV tPA if they meet the criteria. IAT may be offered to these patients with a clear understanding that the standard of care in AIS in $<3$-h duration is IV tPA administration. Those patients who do not meet the IV tPA criteria due to time window or some other factors may be offered IAT, preferably in the setting of a randomized or prospective trial [7]. Intravenous tPA has been recently shown to be safe in a subset of stroke patients to a maximum of $4.5 \mathrm{~h}$ from symptom onset with an $8 \%$ benefit in increasing the probability of good outcomes [9, 10]. Patients with large vessel occlusions [11, 12] and those with large thrombus burden (i.e., thrombi longer than $8 \mathrm{~mm}$ ) [13], and those with more severe strokes respond less well to IV thrombolysis [14]. For these patients, IAT may be considered as an alternative to IV thrombolysis, although currently IV tPA is the only validated approach and its use is recommended to a maximum of $4.5 \mathrm{~h}$ from stroke onset [10]. In 1 observational study of 112 patients with a hyperdense middle cerebral artery sign (MCA), in which half the patients received IV and the other half received IAT, a favorable outcome was doubled and the risk of death was reduced by $2 / 3$ in patients treated with IAT [15]. However, such provocative studies merely emphasize the need for a randomized trial comparing IV therapy with endovascular therapy.
The traditional time window for IAT is $<6 \mathrm{~h}$ and for mechanical embolectomy is $8 \mathrm{~h}$ from stroke onset or the last time the patient was known to be normal [7]. The duration of ischemia is a leading predictor of neurological outcome, but with modern penumbral imaging it may be that time alone may not be a necessary arbiter of who qualifies for IAT [1618]. The author (Abou-Chebl [19]) and his colleagues have treated highly-selected patients well beyond these time limits with excellent success, but such treatment is not the standard of care, and it is controversial and considered experimental [20].

All patients should have the standard clinical, laboratory, and radiological testing before an intervention is contemplated. Patients must also have a clinical deficit severe enough to warrant intervention both to avoid the risk of the procedure and to ensure that an intervention is likely to be of benefit, because patients with mild strokes (National Institutes of Health Stroke Score $[\mathrm{NIHSS}]<4$ ) are unlikely to have a visible arterial occlusion and are likely to have a good outcome without therapy [21]. In contrast, patients with the most severe strokes (NIHSS $>20$ ) are less likely to benefit from treatment although penumbral imaging to identify patients with small ischemic cores and large perfusion deficits may help select who may benefit from treatment [16-18]. It is important to note that such approaches remain unproven. There are several contraindications to AIS intervention, the primary being any history or propensity for ICH (Table 1).

As previously discussed, patients are often selected for IAT with the help of perfusion imaging. These techniques permit the assessment of the ischemic penumbra and may define any salvageable brain tissue and the size of the necrotic, irreversibly injured core. The ideal patient is the 1 with little to no necrotic core and a large volume of penumbra $[16,17,21]$. These patients may have the most to gain with the least risk in contrast to patients with a large necrotic core, who even with concurrent large penumbra
Table 1 Contraindications to Acute Ischemic Stroke Intervention

${ }^{a}$ Unruptured, incidental, nonthrombosed aneurysms are not a contraindication

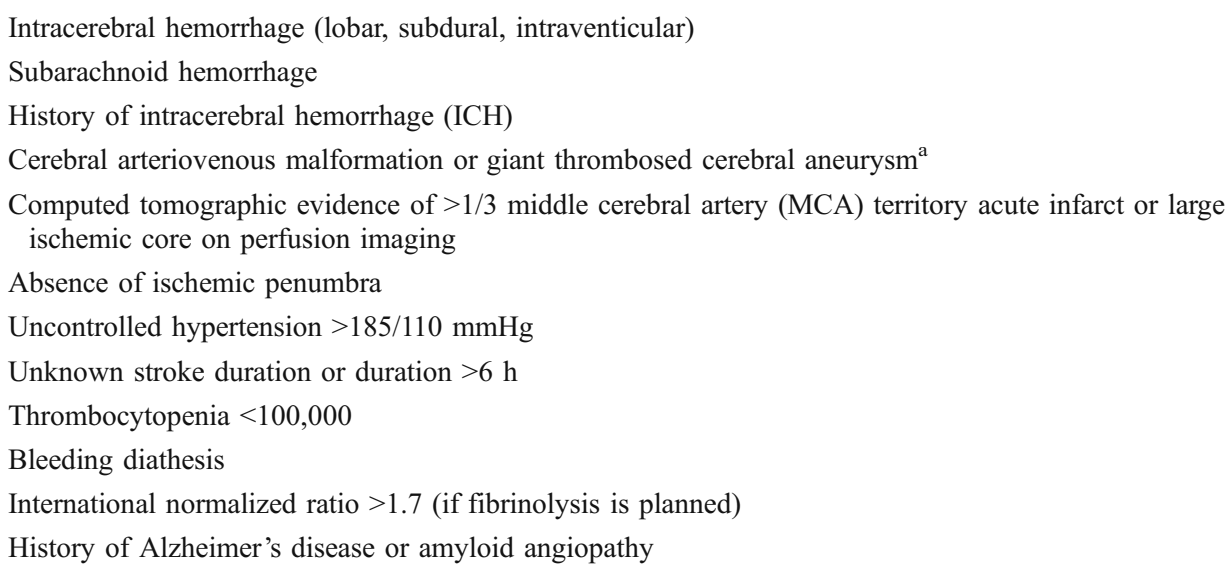


have high ICH risk because revascularization of necrotic tissue is one of the presumed mechanisms of hemorrhagic transformation; the size of the necrotic core may be the best predictor of outcomes [21]. Those patients with no penumbra, even with no necrotic core, in general, may have little to nothing to gain by IAT and would be exposed to unnecessary risk with treatment. The penumbra and ischemic core can be assessed noninvasively with magnetic resonance and computed tomographic (CT) imaging (Fig. 1). However, there is no consensus on the exact definition of penumbra, which technique best defines it, and importantly no validation of the clinical usefulness of such imaging modalities; therefore, until clear cut data emerge that 1 definition or modality is superior to the other, clinicians should use whatever definitions and modalities for which they have the most experience and that have been validated at their institutions.

A noncontrast CT of the head remains the standard of care, and it is the only study recommended by the published guidelines [7]. A potentially simpler approach than perfusion imaging (albeit 1 that requires further validation) is to compare the presence of early infarct signs on $\mathrm{CT}$ with the severity of the neurological deficit. If a CT-clinical mismatch is found (i.e., the CT changes are relatively minor compared to the deficit), then therapy may be initiated. The best validated means of determining "minor" CT scan changes is to use the Alberta Stroke Program Early CT Score (ASPECTS). A score of $>7$ is associated with good neurological outcomes in general, as well as following IAT [22]. There are some conflicting data on the validity of this technique, but its appeal is its simplicity, rapidity, and safety in obviating the need for X-rays and contrast [23].

In addition to parenchymal imaging, vascular imaging by either CT angiography or magnetic resonance angiography can be very helpful in deciding which patients should be offered IAT and how they should be treated. For example, patients without obvious large vessel occlusion, but with a clinical syndrome that suggests large vessel ischemia, may have multiple distal cortical branch occlusions that may not be amenable to mechanical recanalization techniques, but may be better treated with IV thrombolysis or intra-arterial thrombolytics. In addition, such patients may have seizures or other nonstroke causes of the deficits and may not need IAT. On the other hand, the presence of complete internal carotid artery (ICA) occlusion from the bulb to the terminus with MCA occlusion and no pial collateral flow from the anterior cerebral artery is a predictor of poor response to IV thrombolysis, even in patients treated in $<3 \mathrm{~h}[12,24]$. Even IAT has a relatively low probability of complete recanalization (compared to MCA branch occlusion, for example) in such patients, and when taken into consideration with other mitigating factors, such as advanced age, hypertension, and prolonged duration of ischemia, ICA terminus occlusion portends a poor prognosis regardless of recanalization method [2527]. By comparison, patients with cervical ICA occlusion, with or without tandem MCA occlusion have a better prognosis than those with ICA terminus occlusion $[28,29]$. Other findings, such as the presence of direct (i.e., communicating arteries) or pial collaterals, the status of the other vessels, the presence of severe tortuosity, aneurysms, and so forth, are all important to glean from the noninvasive imaging and can save valuable time during the intervention by obviating the need to perform angiograms of the other vessels or by alerting the interventionist for the need to access the vessel in question with more supportive catheters and devices from the outset.

\section{Clinical Aspects}

It may be valuable to determine the likely etiology of the stroke prior to the initiation of IAT. A lesion likely to be due to atherosclerotic occlusion can be approached differently than the 1 due to cardioembolism (e.g., the former may be directly stented and the latter approached first with an embolectomy device) [30]. This approach of tailoring the intervention to the presumed mechanism of occlusion has not been validated, and most studies have not taken the etiology into consideration. Other factors, such as age of the patients, duration of ischemia, presence of early infarct signs, presenting blood pressure, serum glucose level, underlying dementia, and the presence of collaterals are also considered [7, 19, 31, 32]. Based on these clinical and imaging factors the endovascular approach can be planned.

\section{Peri-procedural Medical Management}

It has been common practice to perform IAT with the aid of general anesthesia (GA). Most patients with AIS are able to breathe spontaneously and should not have GA and endotracheal intubation (patients with diffuse brainstem ischemia notwithstanding). The induction of GA and mechanical ventilation may delay revascularization and more importantly can mask spontaneous or therapy-induced neurological recovery or deterioration. If the former occurs, which it does in a small subset of patients, then IAT may no longer be indicated. The cerebral vessels are densely innervated and any excessive dilation or mechanical forces applied to them often result in patient discomfort. The astute interventionist can use this information to reassess device sizing, wire tip position, and so forth, and make appropriate changes. Others have argued that IAT should be performed under GA to make the procedure safer by avoiding inadvertent wire perforation due to patient motion. A recent study by the author (Abou-Chebl [33]) and his colleagues casts doubt into the validity of these concerns. In 
a pooled analysis of 980 consecutive patients from 12 medical centers receiving IAT, those treated under GA had a greater than 2-fold risk of poor outcomes and a nearly 2fold risk of death [33]. Importantly, those treated while awake did not have any increase in the risk of ICH or wire perforation. Although these data require validation, the sheer size of the cohort, 1 of the largest ever published of IAT, suggests that the burden of proof rests with those advocating GA to prove that it is needed, because there are no prospective data that show its benefits.

Blood pressure control is the most important periprocedural clinical factor. Augmentation of low to normal blood pressures or induced hypertension are controversial issues that need validation, however, the lowering of blood pressure in the setting of AIS is less controversial. The American Heart Association (AHA) recommends not to treat systolic blood pressure acutely unless it is above $220 \mathrm{mmHg}$, and in those receiving thrombolytics, the MAP should be kept to $<135 \mathrm{mmHg}(<185 / 110 \mathrm{mmHg})$ [7]. After complete recanalization, blood pressure should be lowered to the normal or below normal ranges because prevention of ICH is the single most important duty at this point. This has also not been validated as an approach. In cases of incomplete recanalization, some mild degree of hypertension may be desired to maintain collateral flow to the nonperfused regions. If ICH is observed, suspected, or believed to be likely, immediate lowering of blood pressure should be performed.

\section{Postoperative Management}

Blood pressure control as previously discussed is critical. After successful intervention, there is no clear indication for continuing a heparin drip or glycoprotein IIb/IIIa (GPIIb/ IIIa) antagonists. Although the Prolyse in Acute Cerebral Thromboembolism II (PROACT II) study showed that a 4$\mathrm{h}$ drip of unfractionated heparin $(500 \mathrm{U} / \mathrm{h})$ may be of benefit, there are no data supporting its use after the infusion of other thrombolytics (i.e., recombinant prourokinase), the thrombolytic used in the PROACT trials requires heparin for optimal fibrinolysis [20]. Generally, other antithrombotics should not be given for at least $24 \mathrm{~h}$ after IAT, much like the standard practice after IV tPA [7]. When a stent is placed, however, aspirin and clopidogrel may be given weighing the risk of ICH $v s$ stent thrombosis. There are no good data to provide a guide to practice here, and every patient situation tends to be unique; however, the general rule of thumb should be that it is always easier to add antithrombotics than to take their effects away.

Postoperative neurological checks should be frequently performed. The occurrence of any headache, with or without worsening of neurological status, should be considered a possible sign of ICH warranting immediate clinical evaluation and emergent CT scan of the brain. The reversal of all antithrombotic and thrombolytic agents should be carried out immediately, if an ICH is found, and emergent neurosurgical consultation should be considered, despite the fact that it is unclear whether or not any of these interventions, including neurosurgery, are of any clinical benefit [34].

\section{Interventional Approach}

Access is obtained rapidly via the femoral artery. Stable access to the culprit vessel can be achieved with a 6-French guide catheter and a short femoral sheath, but in cases of marked tortuosity, placement of a long (typically $80-\mathrm{cm}$ ) No. 6- to 8-French sheath in the corresponding common carotid or subclavian artery is recommended. Prior to sheath or guide placement in the carotid or subclavian a $2,000 \mathrm{U}$ bolus of heparin is given, followed by a $500 \mathrm{U} /$ $\mathrm{h}$ infusion or periodic boluses of 500 to $1,000 \mathrm{U}$. Higher doses may be used, but with greater risk of ICH [20].

A variation of this approach is to place a balloon occlusion guide catheter as it may facilitate mechanical embolectomy by allowing aspiration through the guide and occlusion of antegrade blood flow. This approach can increase the risk of arterial dissection and the available guide catheters (from Concentric Medical Systems Inc.) are not curved and are not as stable as the conventional guide catheters.

\section{Intra-arterial Thrombolysis}

The intra-arterial infusion of thrombolytics directly at the site of the occlusion has been used for more than 20 years in the treatment of AIS [35]. There have been numerous case reports and small case series [35-39], but only 2 significant randomized trials. The first was the Prolyse in Acute Cerebral Thromboembolism II (PROACT II) trial, a phase 3 trial designed to assess the clinical efficacy and safety of $9 \mathrm{mg}$ of IA pro-urokinase (pro-UK) in angiographically documented MCA occlusion of $<6$-h duration [20]. In this study, 180 patients were randomized 2:1 to receive either IA rpro-UK plus $4 \mathrm{~h}$ of low-dose IV heparin or low-dose IV heparin alone. The rpro-UK and control groups were wellmatched, except for a higher prevalence of diabetes among the control patients $(13 \%$ vs $31 \%)$ and more European Cooperative Acute Stroke Study (ECASS) CT scan protocol violations among rpro-UK patients $(10 \%$ vs $4 \%)$. The median pretreatment NIHSS score was 17 and the median time to initiation of treatment was $5.3 \mathrm{~h}$. IA rpro-UK was infused for a 2-h period, even if recanalization was achieved earlier, and no mechanical disruption of the clot was permitted. Control subjects received an angiogram and infusion of heparin. The 2-h partial or complete recanalization (Thrombolysis in Myocardial Infarction [TIMI] 2 or 3) 
rate was $66 \%$ in the treatment group and $18 \%$ in controls, but complete recanalization (TIMI 3) was seen in only $19 \%$ and $2 \%$, respectively. The primary endpoint, the proportion of patients with slight or no disability at 90 days (Modified Rankin Scale [mRS] score $\leq 2$ ), occurred in $40 \%$ of the treatment group of patients and $25 \%$ of controls. This was an absolute benefit of $15 \%$, but a relative benefit of $58 \%$ with a number needed to treat of $7(p=0.043)$. This benefit was greatest in patients with mild and moderate strokes (NIHSS 11-20), those younger than age 68, those who had early infarct volume $<5.25 \mathrm{~mL}$, and those with an ASPECTS score of $>7[22,40]$. The benefit came at a cost of increased symptomatic ICH with a rate of $10 \%$ in the treatment group compared to $2 \%$ in controls at $24 \mathrm{~h}$. These data were insufficient for FDA approval and therefore the only drug proven to be effective with IA infusion is not commercially available; however, this trial proves that IAT, at least with rpro-UK, is safe and effective.

The second major randomized trial was the Middle Cerebral Artery Embolism Local Fibrinolytic Intervention Trial (MELT) [41]. As with the PROACT II trial, the MELT trial evaluated the safety and efficacy of intra-arterial urokinase in patients with M1 or M2 segments of the MCA occlusions of $<6$-h duration. An IA infusion of $120,000 \mathrm{U}$ of urokinase was given for $5 \mathrm{~min}$ and repeated until the total dose reached $600,000 \mathrm{U}, 2 \mathrm{~h}$ had passed, or complete recanalization was achieved. After enrolling 114 patients, the trial was stopped by the steering committee after the approval of IV tPA in Japan. The primary endpoint of $\mathrm{mRS} \leq 2$ was not significantly different between the 57 treatment patients and 57 control patients $(49.1 \%$ vs $38.6 \%$; $p=0.345)$. However, a preplanned secondary analysis showed that the rate of recovery to normal or near normal $(\mathrm{mRS} \leq 1)$ was higher in the treatment group than in the controls $(42.1 \%$ vs $22.8 \% ; p=0.045)$. The rate of ICH was similar to that seen in PROACT II treatment patients $(9 \%)$ and controls $(2 \%)$. Although the numbers from the MELT trial are inconclusive due to the early cessation of the trial, the similarity of the outcomes with PROACT II prove the concept that an open artery improves outcomes and that IAT is an effective treatment in selected patients.

The reality, however, is that in clinical practice, tPA is the most widely used IA lytic, despite the lack of any large randomized trial data and there are reasons to believe that not all lytics are the same (e.g., streptokinase was tested in acute stroke and the trials were stopped due to excessive ICH [42]). Similarly tPA, although proven with IV infusion, may not be the ideal agent for IAT because of some neurotoxic effects resulting in higher rates of ICH [43]. There are multiple reports and small series, some prospective, of the use of other thrombolytics for IAT, including urokinase, reteplase, and tenecteplase, with promising but not definitive results [37, 44]. The optimal dose of each thrombolytic is unknown. As a rule of thumb, however, lower doses are preferred to minimize the risk of ICH taking into account factors that may increase the risk of ICH (e.g., patient age $>80$ years, hypertension $>185 /$ $110 \mathrm{mmHg}$, elevated serum glucose, duration of ischemia, absence of collateral blood flow, underlying infarct size, large clot burden, concurrent use of anticoagulants or other agents, coagulopathy, or aggressive mechanical manipulation during the interventions) [7]. Based on the results of the PROACT II study, a 10\% rate of symptomatic ICH after interventional therapy of AIS is considered acceptable, and this should be the benchmark for all future studies or clinical use of other thrombolytics [20].

Most clinical experience has been done with the slow infusion of a thrombolytic agent for $1 / 2 \mathrm{~h}$ to $2 \mathrm{~h}[20,37$, 38]. In the authors' opinion, waiting $2 \mathrm{~h}$ for a fibrinolytic to take effect is too long; more rapid recanalization techniques should be considered (e.g., multimodal therapy or mechanical clot extraction) particularly if the thrombus burden is high. If distal branch occlusions are the problem, microcatheter fibrinolytic infusions are appropriate, and they are more likely than M1 or BA occlusions to recanalize quickly in this way [26]. Depending on the clinical circumstances (see as follows), the dosage and rate of infusion can be adjusted with no clear consensus on the optimal technique. The total dose of fibrinolytic is generally $1 / 4$ to $1 / 5$ of the intravenous dose, and it is infused primarily within and just proximal to the thrombus; however, some advocate infusion of a small amount of lytic immediately distal to the thrombus $[27,35,36,38]$. More data are needed before any definitive statements can be made regarding the clinical efficacy and safety of the intra-arterial infusion of commercially available thrombolytics.

\section{Multimodal Approach}

Increasingly, the combination of both thrombolytic agents and GPIIb/IIIa antagonists has been described in small series and case reports [30, 45]. There have also been 2 small prospective feasibility studies of combination thrombolysis and GPIIb/IIIa receptor antagonists. The first was a 26 patient study with 16 patients receiving IA urokinase and 10 patients receiving a combination of IA urokinase and IV abciximab [39]. The combined group required $1 / 2$ the dose of urokinase $(418,000 \mathrm{IU} v s 828,000 \mathrm{IU} ; p<0.005)$ to achieve recanalization with no difference in symptomatic $\mathrm{ICH}$, although it was high in both groups, $30 \%$ and $25 \%$, respectively. The other trial was a dose-ranging study of 20 patients, 18 of whom received IA reteplase and IV abciximab [46]. There was 1 symptomatic $\mathrm{ICH}$, and recanalization was achieved in 13 of 20 patients $(65 \%)$. In both studies there were trends toward good outcomes. These agents have been proven safe and effective in the setting of acute myocardial infarction, a disease associated 
with occlusive platelet-rich thrombi. The safety and efficacy of endovascular treatment of acute ischemic stroke remains uncertain; however, these agents may be considered in selected patients, and these seem to make sense in cases of atherosclerotic occlusions or artery-to-artery emboli, but less so in the setting of cardioembolism in which fibrin rich clots are expected. Another potential role of the GPIIb/IIIa antagonists is following emergency stent implantation to prevent acute stent thrombosis. However, because the fundamental principle of AIS therapy is rapid and timely, revascularization with the least risk of $\mathrm{ICH}$ when adding GPIIb/IIIa receptor antagonists to either fibrinolytics or angioplasty and stenting procedures, the neurointerventionist must weigh the definite increased risk of ICH vs the potential benefit. In addition, because there are no randomized trial data that have proven the safety and efficacy or guided the choice, route, and dosage of GPIIb/IIIa receptor antagonists, the safest approach is to avoid these drugs, but if they must be used, then the smallest possible dose of the shortest acting agent should be used, and they should be avoided in high-risk patients (see clinical aspects). There is no indication for continuous infusion of these agents, or any fibrinolytic, or antithrombotic drug (even heparin) for that matter, after the procedure, because prolonged infusions are associated with very high rates of $\mathrm{ICH}$ and death [7, 47].

\section{Combined IV/IA Thrombolysis}

IV thrombolysis can be initiated much more quickly than IAT in most circumstances, because the latter requires the availability of an interventional suite as well as an interventional team who may or may not be available or in the hospital when the decision is made to treat. Even after the patient and team are ready, arterial access still needs to be obtained, and then the vessel in question and finally the occlusion have to be reached with the appropriate endovascular tools. All of these steps may be delayed or prolonged for a multitude of reasons, including difficult femoral arterial access due to morbid obesity or peripheral arterial disease and/or difficult access to the cerebral vasculature due to severe tortuosity in the aorta, great vessels, ICA, and so forth. Infrequently, hours may be spent before a single therapeutic intervention can be initiated. Therefore, it stands to reason that if IV therapy can be initiated quickly, then although the IAT team is preparing and access is obtained, the thrombolytic may begin "chewing up" the clot. This approached has been addressed by several small reports, but the most rigorous data come from the Interventional Management of Stroke (IMS) Study I and Study II.

The IMS Study I was a multicenter, open-label, singlearm pilot study of the feasibility and safety of a combined IV and IA approach to recanalization in 80 patients with ischemic stroke (ages, 18-80, with mean NIHSS 18 \pm 4.5 ). The patients received lower dose IV tPA $(0.6 \mathrm{mg} / \mathrm{kg}, 60 \mathrm{mg}$ maximum, with $15 \%$ as a bolus and the remainder infused for $30 \mathrm{~min}$ ). Patients then underwent cerebral angiography, and if a thrombus was identified, then additional tPA was infused at the site of the thrombus to less than a total dose of $22 \mathrm{mg}$ until complete recanalization was achieved or a maximum of $2 \mathrm{~h}$ of IAT or $7 \mathrm{~h}$ had elapsed because of symptom onset. A total of 62 of the 80 patients received IA tPA on average approximately $81 \mathrm{~min}$ after the initiation of IV therapy [48]. These patients were compared with the patients in the National Institute of Neurological Disorders and Stroke (NINDS) tPA Stroke Trial. Patients who achieved $m R S \leq 2(43 \%)$ and had a 90-day mortality rate in the IMS Study I was 16\% (numerically lower, but not statistically different from the mortality rates of $24 \%$ and $21 \%$, respectively, of the placebo or tPA-treated patients from the NINDS tPA Stroke Trial). The rate of symptomatic ICH was $6.3 \%$, similar to that of the IV tPA-treated patients [49]. Recanalization efficacy was slightly lower than what was noted in the PROACT II trial with the rate of complete recanalization (TIMI 3) of $11 \%$ and partial or complete recanalization (TIMI $2-3$ ) rate of $56 \%$.

The IMS II trial objective was to assess the feasibility of the combined IV and IA approach with the aid of the MicroLysUS infusion catheter (EKOS Inc., Bothell, WA). This catheter is able to deliver ultrasonographic energy with the aim of increasing the permeability and penetration of thrombolytics. The thrombolysis protocol was otherwise the same as that used in IMS Study I, and patients who had to have an occlusion of 1 of the MCA segments (M1 or M2), vertebral artery, or basilar artery (BA). Again, primary comparisons were made with similar subsets of placebo and tPA-treated patients from the NINDS tPA Stroke Trial, as well as those from IMS Study I. From 81 enrolled patients, there were 55 patients who received both IV and IA tPA via either the EKOS catheter $(N=36)$ or a standard microcatheter $(N=19)$ [50]. A good neurological outcome $(\mathrm{mRS} \leq 2)$ was achieved in $46 \%$ compared with $39 \%$ and $28 \%$, respectively, of the patients in the tPA and placebo arms of the NINDS IV tPA Stroke Trial. After an adjustment for baseline factors (NIHSS, age, and time to treatment), the odds ratio (OR) of attaining $\mathrm{mRS} \leq 2$ were 1.74 (95\% CI, 0.95-3.19) and 2.82 (95\% CI, 1.54-5.16), respectively, compared with tPA- and placebo-treated subjects. The 90-day mortality rate in the IMS Study II (16\%) was numerically identical to that noted in the IMS Study I as was the rate of symptomatic ICH $(9.9 \%)$. The recanalization rate for the 55 patients treated with IV plus IA was 4\% Thromolysis In Cerebral Ischemia (TICI)/TIMI 3 and $60 \%$ Thromolysis In Cerebral Ischemia (TICI)/TIMI 2 to 3 reperfusion, but for the subset treated with the EKOS catheter complete recanalization was noted in $41.4 \%$ and 
TIMI 2 or 3 recanalization was noted in $62.0 \%$ [51]. Of note in a subsequent analysis of 75 of 81 patients in IMS Study II with anterior circulation strokes, the use of heavy sedation/pharmacologic paralysis was associated with a markedly increased risk of poor clinical outcomes compared to those treated with no to mild sedation (OR, $7.0 ; 95 \% \mathrm{CI}$, 2.0-24.5; $p=0.002$ ), including a significantly higher risk of death (OR, 5.0; 95\% CI, 1.34-18.7; $p=0.02$ ) [52].

In 1 retrospective study at a single institution of 107 patients treated with IV tPA for large vessel occlusion compared with 53 patients treated with IV tPA followed by endovascular therapy, recanalization was achieved in $87 \%$ of the IVendovascular treatment group $v 552 \%$ in the IV group (adjusted relative risk [RR], 1.49; 95\% CI 1.21-1.84; $p=$ 0.0002) [53]. Early neurological improvement (NIHSS score of 0 or 1 , or an improvement of 4 points or more at $24 \mathrm{~h}$ ) occurred in $60 \%$ of patients in the IV cendovascular group and $39 \%$ of patients in the IV group (adjusted RR, 1.36; CI 0.97-1.91; $p=0.07)$. A good outcome (mRS $\leq 2$ at 90 days) occurred in $57 \%$ of patients in the IV endovascular group and $44 \%$ of patients in the IV group (adjusted RR, 1.16; CI $0.85-1.58 ; p=0.35)$. The mortality rate at 90 days was $17 \%$ in both groups, and symptomatic ICH was $9 \%$ and $11 \%$, respectively.

In clinical practice there has been widespread adoption of the IMS Study low-dose IV tPA protocol or so-called "bridging dose," in which the results of the trials previously discussed are less than definitive with clinical outcomes and mortality rates either slightly better or no better than IV tPA alone. Therefore, currently there are no data to support the routine administration of low-dose IV tPA given to patients who otherwise qualify for full-dose IV tPA treatment with the intent of then initiating IAT. Until the results of the IMS Study III (i.e., the phase 3, definitive, comparative efficacy study of the "bridging dose" approach), the guidelines recommend full-dose IV tPA administration to all patients who qualify [7]. Then, if patients "fail" full-dose thrombolysis, rescue IAT may be considered (see as follows). Here again there is no agreed definition of failure, and when it is appropriate to treat patients who have received full-dose IV tPA with IAT. In a recent study using transcranial Doppler ultrasound in 179 patients treated with IV tPA, Ribo et al. [54] documented that the majority of recanalization occurs within the first hour with few recanalization afterwards. These data suggest that if patients do not clinically improve and recanalize within $1 \mathrm{~h}$, as assessed by noninvasive imaging (transcranial Doppler ultrasound, magnetic resonance angiography, or CT angiography), they may be candidates for IAT. The embolectomy trials that included patients with failed IV thrombolysis did not define failure other than that tPA "failed to open the intracranial large vessel as proven by conventional angiography" [55].

\section{Mechanical Clot Disruption and Extraction}

Mechanical approaches to arterial recanalization using balloons, snares, or the embolectomy devices have been reported with promising results [56-58] (Fig. 2). Angioplasty, especially of underlying atherosclerotic or stenotic lesions, has been highly effective in several large Japanese series that reported recanalization rates of $\leq 91 \%$ with lower risks of ICH compared to intra-arterial fibrinolysis ( $3 \%$ vs $>10 \%)[59,60]$. This degree of angioplasty success may be unique to the Japanese populations studied because they have a high incidence of intracranial atherosclerosis, as the cause of ischemia unlike the lower incidence of 8 to $10 \%$ in the United States [61, 62]. However, angioplasty is sometimes effective, even without an underlying stenosis $[30,63]$. Angioplasty alone does carry a risk of acute and delayed re-occlusion, particularly if there is an underlying stenosis. There are insufficient data on the frequency of this event in the setting of acute ischemic stroke, although it has been described to occur $16 \%$ of the time in 1 series of elective angioplasty for intracranial atherosclerotic disease [64], but $0 \%$ of the time in small acute stroke series [65, 66]. In the treatment of acute ST segment elevation myocardial infarction, balloon angioplasty alone is associated with abrupt closure 2.3 to $5 \%$ of the time with an approximately $10 \%$ ischemic event rate (i.e., reinfarction, death, or stroke) $[67,68]$. Clearly more data are needed on the safety and efficacy of this approach.

Anecdotally, it seems that stenting of an underlying stenosis or occlusion is almost always effective in achieving recanalization (although it is not necessarily reperfusion) and may be considered in selected patients $[27,30,69,70]$. A recent report of a prospective study of 20 patients with AIS treated with the Wingspan stent (Stryker Inc., Fremont, CA) highlights the potential of this technique [71]. The mean NIHSS of the patients was $14 \pm 3.8$ (median, 13), which was low compared to the previously discussed trials. The patients had to have stroke $<8$-h duration, NIHSS $\geq 8$, angiographicaly documented occlusion of $\leq 14$-mm length, with a parent vessel diameter of 2 to $4.5 \mathrm{~mm}$, with $<1 / 3$ of "at-risk territory ... nonsalvageable" by CT Perfusion (CTP). The important finding was TIMI $2(40 \%)$ or $3(60 \%)$ combined recanalization rate of $100 \%$. The 30 -day rate of $\mathrm{mRS} \leq 3$ was for $60 \%$ of patients and $m R S \leq 1$ at 30 days for $45 \%$ of patients. There was only $1(5 \%)$ symptomatic ICH. The data need to be validated in a larger trial that includes a study of long-term followup, including the risk of re-stenosis. Stenting may be particularly effective for atherosclerotic, acute cervical ICA-origin occlusions, but it comes at a cost of potentially increased risk of complications and the need for dual antiplatelet therapy [28, 70]. The latter are potentially hazardous in individuals who have large infarcts or receive fibrinolytic therapy because of the increased risk of 
ICH; also if the patient were to develop a complete MCA infarct with brain swelling and herniation, then the presence of dual antiplatelet agents may increase the risk of complications with potentially lifesaving decompressive hemicraniectomy. Acute stenting is also associated with high early in-stent restenosis rates (unpublished data). Also, stenting at bifurcations, where emboli tend to lodge, can result in recanalization of one branch but not others. Therefore, stenting remains an unproven therapy with many outstanding questions and it should be used judiciously, if at all, in the setting of acute ischemic stroke.

Mechanical embolectomy with the Mechanical Embolus Removal in Cerebral Ischemia (MERCI) Clot Retriever (Concentric Medical Systems Inc.) was approved by the FDA in August 2005 for the removal of "blood clots from the brain in patients experiencing an ischemic stroke" [57]. With the aid of negative pressure applied through a balloon occlusion guide catheter, the device can be used to extract clots with a 45 to $58 \%$ efficacy. The single arm study of the device in 151 patients with AIS of 3- to 8-h duration, without penumbral imaging, showed feasibility and safety of mechanical embolectomy. The mean age was 67 for the patients in the MERCI study, and the patients had a mean NIHSS of $20.1 \pm 6.6$ with a mean time from symptom onset to groin puncture of $4.3 \pm 1.7 \mathrm{~h}$ [57]. The intervention lasted an average $2.1 \pm 1.0 \mathrm{~h}$. The patients in this study had significantly larger clot burden than those studied in the PROACT Study II and the MELT, and we can assume larger thrombi than in the NINDS IV tPA trial, given the severity of the strokes. Occlusions were noted in the ICA (19\%), ICA terminus (14\%), M1 or M2 segments of the MCA branches $(57 \%)$, intracranial vertebral artery $(1 \%)$, and BA $(9 \%)$. TIMI 2-3 recanalization was achieved in 46\% (69 of 151) of the patients in the intention-to-treat analysis. Adjunctive thrombolysis was permitted achieving a recanalization rate of $60.3 \%$. The overall rates of good outcome ( $\mathrm{mRS} \leq 2)$ and mortality at 90 days were $27.7 \%$ and $43.5 \%$, respectively. Symptomatic ICH was noted in 11 of 141 (7.8\%) patients. Perhaps the most important finding from this study was that successful revascularization was the strongest predictor of good neurological outcome (OR, 12.82; 95\% CI, 2.95$55.75 ; p=0.0001)$. Other factors such as age (OR, $0.94 ; 95 \%$ CI, $0.90-0.98 ; p=0.0009)$ and baseline NIHSS score (OR, 0.78 ; 95\% CI, $0.67-0.89 ; p=0.0007)$ were also associated with good 90-day outcomes. The absence of recanalization, higher age, and higher NIHSS scores were independently associated with 90-day mortality.

The follow-up study, the Multi MERCI trial was an international, multicenter, single-arm trial with plans to obtain further safety data of the first generation Merci Retrievers (X5 and X6), explore the safety and technical efficacy of the devices in patients who "fail" IV thrombolysis, and collect safety and efficacy data on the second- generation Retriever (L5) [55, 72]. A total of 177 patients were enrolled in the Multi MERCI trial, but the device was deployed in only 164 patients, with a mean age of $68.1 \pm$ 16.0 years. The median NIHSS score was 19 (range, 15$23)$, and the median procedural duration was $1.6 \mathrm{~h}$ (1.2$2.3 \mathrm{~h})$. As in the MERCI trial, the patients had a high thrombus burden with ICA terminus occlusion (32\%), M1 or M2 segments of the MCA branch occlusion $(60 \%)$, and posterior circulation occlusion (8\%). There were 48 (29.3\%) patients who had "failed" IV tPA and 57 (34.8\%) patients who received intraprocedural IA lytics. Treatment with either Retriever alone resulted in successful (TIMI 23) recanalization in $55 \%$, with adjunctive thrombolysis in $68 \%$ of vessels that were recanalized. The rates of good outcome (36\%) and mortality (34\%) at 90 days were better than the rates observed in the MERCI trial. The rate of symptomatic ICH was $9.8 \%$, which was similar between those who received IV tPA and those who did not receive

Fig. 1 A 49-year-old man presented within the 3-h window of time for intravenous (IV) tissue plasminogen activator (tPA) with a right middle cerebral artery syndrome and National Institutes of Health Stroke Score (NIHSS) score of 14 with dense hemianopsia, hemiplegia, and sensory loss, but minor neglect and no eye deviation. After IV tPA, the patient had no clinical improvement, and therefore he was taken for endovascular intervention. (a) Pre-tPA computed tomographic perfusion cerebral blood flow, (b) cerebral blood volume, and (c) time to peak images indicating that there was no necrotic core, but a marked delay in perfusion of the entire right middle cerebral artery (MCA) and Posterior Cerebral Artery (PCA) territories. (d) Right internal carotid artery angiogram reveals RICA occlusion (arrow). (e) A left intracerebral hemorrhage (ICH) injection shows a flow across the anterior communicating artery supplying collateral flow to the right Anterior Cerebral Artery (ACA) and MCA. Two large branch occlusions were noted at the M2-M3 level on the right, and these were believed to be the culprit lesions causing the symptoms. The CT Perfusion showed reasonably good Cerebral Blood Flow and Cerebral Blood Volume values in the Right Middle Cerebral Artery (RMCA) cortex; therefore, the culprit lesion was unlikely to be the internal carotid artery (ICA) occlusion. (f, g) A microcatheter was advanced into the RMCA across the anterior communicating from the left ICA and microcatheter injection confirmed the presence of the branch occlusions (f, arrow; g, black arrow). (g) The microcatheter was advanced into the occluded segment of the superior division (white arrow) and tPA was infused. (h) The patient then became sleepy and with right gaze deviation. Left ICA injection demonstrated new RMCA occlusion (arrow). (i) RICA injection confirmed distal migration of the thrombus (arrow). (j) RICA injection after the first pass with the Penumbra device (Penumbra Inc., Alameda, CA) noting complete ICA recanalization (arrow) and partial recanalization of the RMCA (arrow). (k) RICA angiography after the second pass with the Penumbra device (Penumbra Inc.) demonstrating complete recanalization of the RMCA. (l) Final RICA lateral angiogram demonstrates recanalization of the majority of the RMCA territory, but persistent occlusion of a posterior division branch (arrow) and the fetal posterior cerebral artery (white arrow). (m) Final cervical RICA angiogram confirming normal ICA flow. (n, o) 24-h RMCA Diffusion Weighted Imaging (DWI) showing patchy but small RMCA and PCA strokes. The patient had significant clinical improvement with only mild residual left hand weakness and left homonymous hemianopsia with a modified Rankin Scale $(\mathrm{mRS})=2$ at discharge 

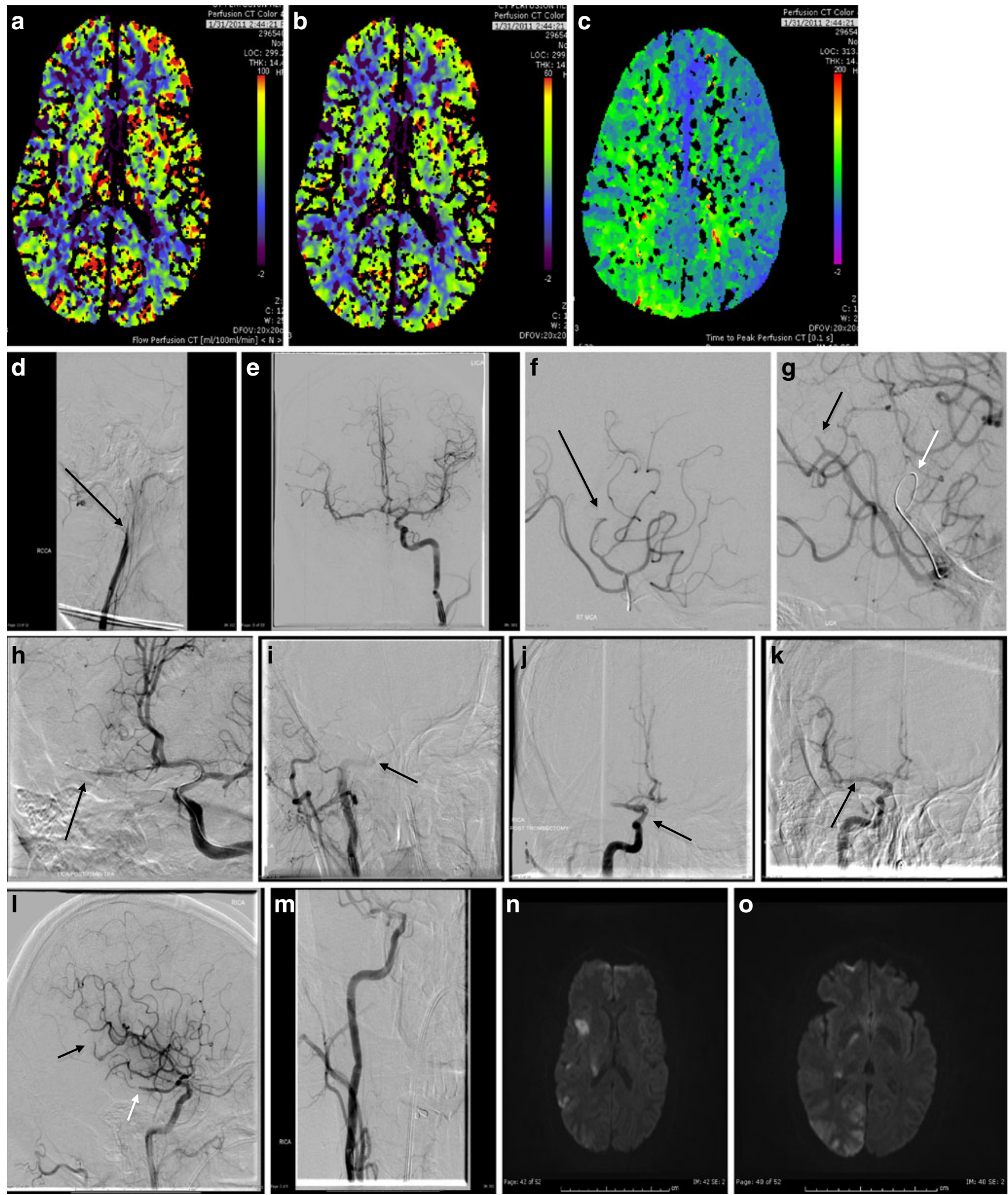

IV tPA $(10 \%$ vs $9.5 \%$, respectively; $p=0.99)$. This study also confirmed that recanalization is the best predictor of good neurological outcomes at 90 days, which was more frequent (49\% vs 9.6\%), and mortality was lower (25\% vs $52 \%$ ) with successful compared with unsuccessful recana-

lization. These trials still did not show unequivocal benefit but this is most likely related to the fact that there is a learning curve with the use of these devices but also the patients enrolled into these trials were far sicker than the patients enrolled in any of the other trials, and they had far 
a

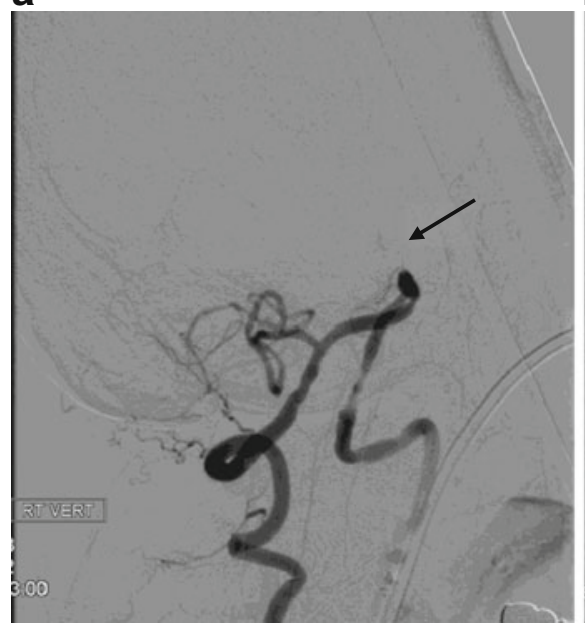

b

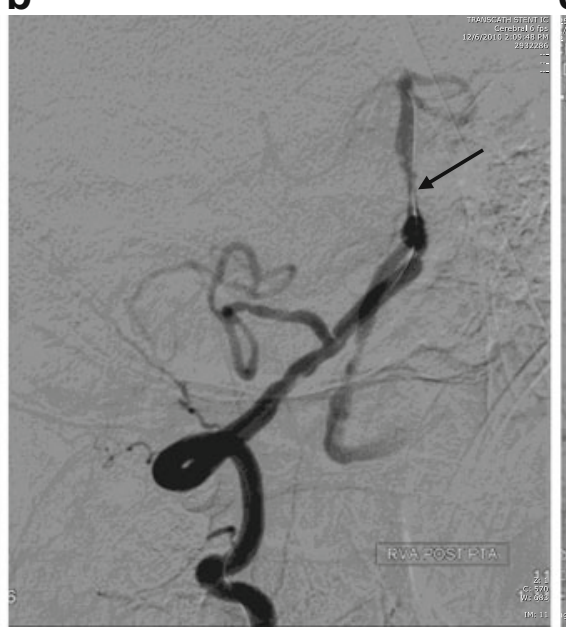

C

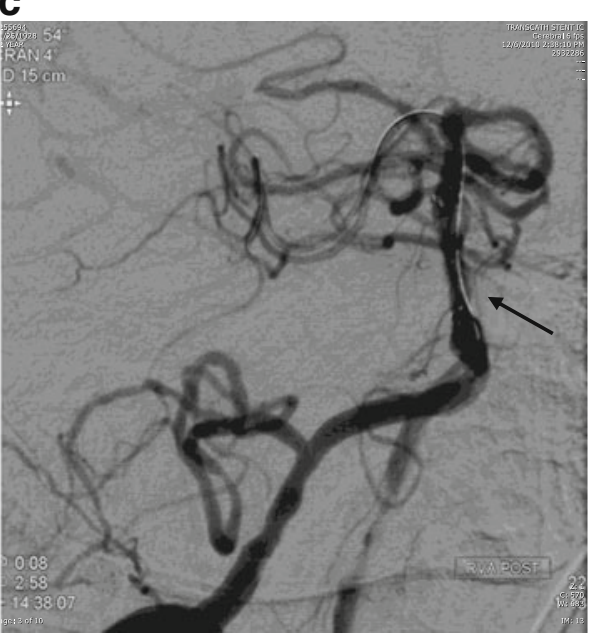

Fig. 2 An 82-year-old man who presented within $3 \mathrm{~h}$ with basilar artery occlusion and received IV tPA with significant improvement. Then $48 \mathrm{~h}$ after the tPA, the patient developed coma and quadriparesis. (a) The angiogram demonstrated recurrent basilar artery occlusion (arrow). (b) After angioplasty with a $2.5 \times 9 \mathrm{~mm}$ balloon, the Basilar Artery (BA) is recanalized, but there was a severe residual proximal
BA stenosis (arrow) with poor distal flow. (c) After $3 \times 15 \mathrm{~mm}$ selfexpanding stent placement and postdilation, the BA and its branches are fully recanalized (arrow). Immediately after the stenting, the patient began to have progressive neurological improvement and is currently residing at home with his family with modified Rankin Scale $(\mathrm{mRS})=3$ longer durations of ischemia. So although mortality rates seem to be higher than the rates seen in the NINDS and other IV thrombolysis trials, we must keep in mind that those trials included patients with lacunar strokes and strokes of lower severity. We have no comparable dataset of patients to be able to conclude that the embolectomy devices (this applies to the discussion of the Penumbra [Penumbra Inc., Alameda, CA] device to follow) caused more harm than good as has been suggested by many. Even in the PROACT II, the study did not include patients with ICA occlusion and patients were treated in $<6 \mathrm{~h}$ from stroke onset. None of the studies conducted with the MERCI Retriever, nor its recent competitor the Penumbra system (Penumbra Inc.) was intended or powered to show efficacy. Therefore, it is a valid argument that these devices are not proven effective in stroke treatment. However, it is clear that recanalization, when appropriately timed, is the most effective treatment for stroke, and these devices do recanalize vessels (Fig. 1). Also, in patients who cannot receive IVor even IA thrombolysis due to coagulopathy or recent surgery, for example, then this approach may be preferred as a reasonable therapeutic option because only low-dose anticoagulation is needed for the procedure.

Recently, data from the largest prospective registry of mechanical embolectomy for AIS were presented. The MERCI Registry included 1,000 patients treated "in the real world" with no exclusion criteria (MERCI Registry, Oral Presentation, ISC February, 2011, Los Angeles Convention Center, Los Angeles, CA). The 90-day follow-up data were available for 872 patients who had a median age of 68 and a median NIHSS of 17 . IV tPA was given to $29 \%$ of patients, and $47 \%$ of patients received additional IA thrombolysis. The mean time from symptom onset to treatment was $6.33 \pm 6.56 \mathrm{~h}$ with $12 \%$ treated $<3 \mathrm{~h}$ and $16.9 \%$ treated $\geq 8 \mathrm{~h}$ from stroke onset. As in the first 2 trials, $32 \%$ (nearly $1 / 3$ ) of the patients had an ICA occlusion, $52 \%$ had MCA trunk occlusion, $8 \%$ had MCA branch occlusion, and $8 \%$ had vertebrobasilar occlusion. Recanalization was achieved in $80.1 \%$ of patients. A good clinical outcome was noted in $31.6 \%$ of patients and mortality was noted in $33.4 \%$. As before, recanalization was the best predictor of good outcome, but there was a decreasing benefit in older individuals. Comparing those $<60$ years of age with those $>79$ years of age, there was approximately a $50 \%$ lower probability of good outcome with recanalization, and if no recanalization was achieved then the probability of good outcome approached $0 \%$ in those $>79$ years of age. As expected, the mortality was highest in those $>79$ years of age who had approximately $40 \%$ mortality, even with successful recanalization, compared to $15 \%$ mortality in those $<60$ years of age. The pattern was similar when outcomes were stratified by presenting the NIHSS score. There was nearly a $70 \%$ probability of a good neurological outcome in those with NIHSS $<16$ who had successful recanalization, but only a $10 \%$ good outcome in those with NIHSS $>25$, even with complete recanalization. Mortality was 10 to $15 \%$ in those patients with NIHSS $<16$, and successful recanalization compared to 40 to $50 \%$ in those with NIHSS $>25$ and successful recanalization. Importantly, the symptomatic ICH rate was as low as $7 \%$ and seemed to be lower in those patients who had successful recanalization $(5.4 \%$ with TICI 3 vs $9.2 \%$ with TICI $0-1)$. In a 
multivariate analysis, the predictors of good neurological outcome $(\mathrm{mRS} \leq 2)$ were at the baseline NIHSS (OR, 0.88 [range, 0.85-0.91]; $p<0.0001)$, age (OR, 0.95 [range, 0.940.97]; $p<0.0001$ ), revascularization (TICI $2 \mathrm{a} / 2 \mathrm{~b} / 3$ ) (OR, 4.02 [range, 2.29-7.08]; $p<0.0001$ ), and intubation during the procedure (OR, 0.41 [range, 0.28-0.6]; $p<0.0001)$.

These data confirm the efficacy of recanalization in improving outcomes. They also suggest that age may play an important role in outcomes and patient selection. IN addition, they also help support the previously presented data in that the use of general anesthesia during AIS endovascular treatment may be deleterious. This last finding must be validated in a prospective and randomized fashion.

In the MERCI registry, 112 patients were treated $\geq 12 \mathrm{~h}$ from stroke onset, and their neurological outcomes were identical to those treated under $8 \mathrm{~h}$ (i.e., the revascularization rate was $81.3 \%$, the 90 -day good outcome rate was $37.8 \%$, and the mortality was $18.8 \%$ ). This data support, but do not confirm, some previously published data on the treatment of patients well beyond the 6-h window [19]. In a study of 55 patients treated at a single institution, 34 were treated with a mean duration of ischemia of $3.4 \pm 1.6 \mathrm{~h}$ and 21 were treated with a mean duration of ischemia of $18.6 \pm$ $16.0 \mathrm{~h}$. The patients had severe strokes with a mean NIHSS of $19.7 \pm 5.7$ and were selected by perfusion imaging, as previously discussed. When comparing the 2 groups, the recanalization rates were similar $(82.8 \%$ vs $85.7 \%$; $p=$ not significant [NS]) as were the rates of good neurological outcome ( $41.2 \%$ vs $42.9 \%$; $p=$ not significant). Importantly, the rates of symptomatic ICH were not higher, even in patients with anterior circulation strokes $(8.8 \%$ vs $9.5 \% ; p=$ NS). As in the MERCI studies, the best predictor of poor outcome and death was the presenting NIHSS, not the duration of ischemia. Taken together, these data suggest that although more study is needed to confirm the results, patients with severe stroke syndromes who also have a poor prognosis without recanalization may be considered for intra-arterial treatment on a compassionate use basis, regardless of duration of ischemia. More study is also needed to validate the selection of patients for IAT, particularly those outside the traditional treatment window, with penumbral imaging $[18,73]$.

The Penumbra clot extraction system (Penumbra Inc.) was the second FDA-approved device for the removal of clots from the brain. It was tested in much the same way as the MERCI system in a single-arm registry with similar inclusion criteria, as well as an 8-h window of time [74]. In this case there was a study of 125 patients, with a mean baseline NIHSS score of $17.3 \pm 5.2$ and a mean age of $63.5 \pm 13.5$ years. The median time from stroke onset to the start of the procedure was $4.1 \mathrm{~h}$ ). The sites of occlusion included the ICA (18\%), M1 or M2 segments of the MCA branches $(70 \%)$, vertebrobasilar arteries $(9 \%)$, and other (3\%). Revascularization (TIMI 2-3) was achieved in $81.6 \%$ of vessels. Good neurological outcomes (mRS score $\leq 2)$ at 90 days were achieved in $25 \%$ of patients with a 90 -day mortality rate of $32.8 \%$. Symptomatic ICH occurred in $11.2 \%$ of patients. As in the MERCI and Multi MERCI trials, there was a trend for good neurologic outcomes to occur $(29 \%$ vs $9 \% ; p=0.0596)$ and mortality rates to be lower $(29 \%$ vs $48 \%$; $p=0.1384)$ with successful compared with unsuccessful recanalization. These results were replicated in a postmarket retrospective review of 139 patients treated with the device at 7 centers with similar recanalization rates and good clinical outcomes in $40 \%$ (Penumbra Post, Oral Presentation, International Stroke Conference, February 20, 2009, San Diego, CA).

There are advantages and drawbacks to each of these devices. One advantage of the Penumbra system in comparison with the MERCI devices is that complete access to the lesion is not always lost, although it is often lost if the clot involves a bifurcation. This is a major benefit in cases with severe proximal tortuosity that would require repeated navigation with the MERCI device (on average, 3 passes are needed to successful recanalization) [72]. Anecdotally, the MERCI system seems to have a lower risk of distal embolization. Both the MERCI and Penumbra devices have been associated with subarachnoid hemorrhage (SAH) and vessel perforation. Great care must be taken when smaller branches (i.e., $<2 \mathrm{~mm}$ in diameter are cannulated and neither device is compatible with vessels $\leq 1.5 \mathrm{~mm}$ ). In practice, most interventionists become familiar with 1 system and use it preferentially, or they will switch from 1 to the other if the first device fails. This is a reasonable approach until a randomized trial shows benefit of 1 device or technique in comparison with another. As a consequence, the interventional treatment of AIS is likely to remain variable between institutions and individual physicians emphasizing the pressing need for prospective data.

A new class of devices is now being tested, the socalled "stentrievers." These devices combine the benefits of stenting with embolectomy devices. One intriguing quality is the ability of the interventionist to deploy the device as a stent, but then recapture it once the thrombus has been lysed, leaving nothing behind. To date, the published data include a small series and 2 prospective pilot trials of 20 and 50 patients each [75-78]. Recanalization efficacy has varied between 80 to $100 \%$ with a symptomatic ICH rate of approximately $10 \%$. Most of the patients treated did not need adjunctive thrombolysis, although in the study by Costalat et al. [78] many patients received thrombolysis. Several manufacturers have developed stentrievers that are in the various stages of testing, although none are commercially available in the United States yet. 


\section{Limitations}

AIS interventions remain essentially "investigational" and unregimented. Much of the previous discussion and much of the literature represent little more than anecdotal experience, albeit somewhat scientifically based. Another major limitation is that recanalization efficacy with traditional intra-arterial fibrinolysis is relatively poor compared with that achieved with acute coronary syndrome interventions, although stenting, mechanical embolectomy, and multimodal approaches are able to achieve nearly complete recanalization. This technical success has not been associated with equivalent improvement in clinical outcomes, and recanalization has not always equated with reperfusion. The reasons for this are not known with certainty, but may included inadequate patient selection, poor technique, lack of operator experience, direct injury from thrombolytics or devices, microvascular occlusions, or some other unknown factors. Intracerebral hemorrhage still complicates 7 to $15 \%$ of procedures. Complete recanalization itself may be responsible for the poor outcomes, either due to ICH or to reperfusion injury and cytotoxicity; therefore, some patients are better off not receiving recanalization therapy. Large prospective and randomized clinical trials are needed to determine the safety and efficacy of IAT, whether it is pharmacological, embolectomy, stenting, or multimodal therapy. Comparative studies with IV tPA will also be needed, especially for the 3 - to 4.5 -h window (the IMS Study III may give us answers for the 3-h window).

Acknowledgment Full conflict of interest disclosure is available in the electronic supplementary material for this article.

\section{References}

1. Albers GW, Olivot JM. Intravenous alteplase for ischaemic stroke. Lancet 2007;369:249-250.

2. Lloyd-Jones D, Adams R, Carnethon M, et al. Heart disease and stroke statistics - 2009 update: a report from the American Heart Association Statistics Committee and Stroke Statistics Subcommittee. Circulation 2009;119:480-486.

3. Wolpert SM, Bruckmann H, Greenlee R, Wechsler L, Pessin MS, del Zoppo GJ. Neuroradiologic evaluation of patients with acute stroke treated with recombinant tissue plasminogen activator. The rt-PA Acute Stroke Study Group. AJNR Am J Neuroradiol 1993;14:3-13.

4. Saqqur M, Uchino K, Demchuk AM, et al. Site of arterial occlusion identified by transcranial Doppler predicts the response to intravenous thrombolysis for stroke. Stroke 2007;38:948-954.

5. de Boer SP, Barnes EH, Westerhout CM, et al. High-risk patients with ST-elevation myocardial infarction derive greatest absolute benefit from primary percutaneous coronary intervention: results from the Primary Coronary Angioplasty Trialist versus thrombolysis (PCAT)-2 collaboration. Am Heart J 2011;161:500-507.
6. Adams HP Jr., Brott TG, Furlan AJ, et al. Guidelines for thrombolytic therapy for acute stroke: a supplement to the guidelines for the management of patients with acute ischemic stroke. A statement for healthcare professionals from a Special Writing Group of the Stroke Council, American Heart Association. Circulation 1996;94:1167-1674.

7. Adams HP Jr., del ZG, Alberts MJ, et al. Guidelines for the early management of adults with ischemic stroke: a guideline from the American Heart Association/American Stroke Association Stroke Council, Clinical Cardiology Council, Cardiovascular Radiology and Intervention Council, and the Atherosclerotic Peripheral Vascular Disease and Quality of Care Outcomes in Research Interdisciplinary Working Groups: the American Academy of Neurology affirms the value of this guideline as an educational tool for neurologists. Stroke 2007;38:1655-1711.

8. Hacke W, Ringleb P, Stingele R. [How did the results of ECASS II influence clinical practice of treatment of acute stroke]. Rev Neurol 1999;29:638-641.

9. Hacke W, Kaste M, Bluhmki E, et al. Thrombolysis with alteplase 3 to 4.5 hours after acute ischemic stroke. $\mathrm{N}$ Engl $\mathrm{J}$ Med 2008;359:1317-1329.

10. del Zoppo GJ, Saver JL, Jauch EC, Adams HP Jr. Expansion of the time window for treatment of acute ischemic stroke with intravenous tissue plasminogen activator: a science advisory from the American Heart Association/American Stroke Association. Stroke 2009;40:2945-2948.

11. Kharitonova T, Ahmed N, Thoren M, et al. Hyperdense middle cerebral artery sign on admission CT scan — prognostic significance for ischaemic stroke patients treated with intravenous thrombolysis in the safe implementation of thrombolysis in Stroke International Stroke Thrombolysis Register. Cerebrovasc Dis 2009;27:51-59.

12. De Silva DA, Brekenfeld C, Ebinger M, et al. The benefits of intravenous thrombolysis relate to the site of baseline arterial occlusion in the Echoplanar Imaging Thrombolytic Evaluation Trial (EPITHET). Stroke 2010;41:295-299.

13. Riedel CH, Zimmermann P, Jensen-Kondering U, Stingele R, Deuschl G, Jansen O. The importance of size: successful recanalization by intravenous thrombolysis in acute anterior stroke depends on thrombus length. Stroke 2011;42:1775-1777.

14. Saver JL, Yafeh B. Confirmation of tPA treatment effect by baseline severity-adjusted end point reanalysis of the NINDS-tPA stroke trials. Stroke 2007;38:414-416.

15. Mattle HP, Arnold M, Georgiadis D, et al. Comparison of intraarterial and intravenous thrombolysis for ischemic stroke with hyperdense middle cerebral artery sign. Stroke 2008;39:379383.

16. Albers GW. Expanding the window for thrombolytic therapy in acute stroke. The potential role of acute MRI for patient selection. Stroke 1999;30:2230-2237.

17. Lansberg MG, Thijs VN, Bammer R, et al. The MRA-DWI mismatch identifies patients with stroke who are likely to benefit from reperfusion. Stroke 2008;39:2491-2496.

18. Davis SM, Donnan GA, Parsons MW. Effects of alteplase beyond $3 \mathrm{~h}$ after stroke in the Echoplanar Imaging Thrombolytic Evaluation Trial (EPITHET): a placebo-controlled randomised trial. Lancet Neurol 2008;7:299-309.

19. Abou-Chebl A. Endovascular treatment of acute ischemic stroke may be safely performed with no time window limit in appropriately selected patients. Stroke 2010;41:1996-2000.

20. Jovin TG, Yonas H, Gebel JM, et al. The cortical ischemic core and not the consistently present penumbra is a determinant of clinical outcome in acute middle cerebral artery occlusion. Stroke 2003;34:2426-2433.

21. Furlan A, Higashida R, Wechsler L, et al. Intra-arterial prourokinase for acute ischemic stroke. The PROACT II study: a 
randomized controlled trial. Prolyse in Acute Cerebral Thromboembolism. JAMA 1999;282:2003-2011.

22. Hill MD, Rowley HA, Adler F, et al. Selection of acute ischemic stroke patients for intra-arterial thrombolysis with pro-urokinase by using ASPECTS. Stroke 2003;34:1925-1931.

23. Kent DM, Hill MD, Ruthazer R, et al. "Clinical-CT mismatch" and the response to systemic thrombolytic therapy in acute ischemic stroke. Stroke 2005;36:1695-1699.

24. Rubiera M, Ribo M, Delgado-Mederos R, et al. Tandem internal carotid artery/middle cerebral artery occlusion: an independent predictor of poor outcome after systemic thrombolysis. Stroke 2006;37:2301-2305.

25. Arnold M, Kappeler L, Nedeltchev K, et al. Recanalization and outcome after intra-arterial thrombolysis in middle cerebral artery and internal carotid artery occlusion: does sex matter? Stroke 2007;38:1281-1285.

26. Barreto AD, Albright $\mathrm{KC}$, Hallevi $\mathrm{H}$, et al. Thrombus burden is associated with clinical outcome after intra-arterial therapy for acute ischemic stroke. Stroke 2008;39:3231-3235.

27. Gupta R, Vora NA, Horowitz MB, et al. Multimodal reperfusion therapy for acute ischemic stroke: factors predicting vessel recanalization. Stroke 2006;37:986-990.

28. Jovin TG, Gupta R, Uchino K, et al. Emergent stenting of extracranial internal carotid artery occlusion in acute stroke has a high revascularization rate. Stroke 2005;36:2426-2430.

29. Malik AM, Vora NA, Lin R, et al. Endovascular treatment of tandem extracranial/intracranial anterior circulation occlusions: preliminary single-center experience. Stroke 2011;42:16531657.

30. Abou-Chebl A, Bajzer CT, Krieger DW, Furlan AJ, Yadav JS. Multimodal therapy for the treatment of severe ischemic stroke combining GPIIb/IIIa antagonists and angioplasty after failure of thrombolysis. Stroke 2005;36:2286-2288.

31. Kim JJ, Fischbein NJ, Lu Y, Pham D, Dillon WP. Regional angiographic grading system for collateral flow: correlation with cerebral infarction in patients with middle cerebral artery occlusion. Stroke 2004;35:1340-1344.

32. Cross DT III, Moran CJ, Akins PT, Angtuaco EE, Derdeyn CP, Diringer MN. Collateral circulation and outcome after basilar artery thrombolysis. AJNR Am J Neuroradiol 1998;19:1557-1563.

33. Abou-Chebl A, Lin R, Hussain MS, et al. Conscious sedation versus general anesthesia during endovascular therapy for acute anterior circulation stroke: preliminary results from a retrospective, multicenter study. Stroke 2010;41:1175-1179.

34. Morgenstern LB, Hemphill JC, III, Anderson C, et al. Guidelines for the management of spontaneous intracerebral hemorrhage: a guideline for healthcare professionals from the American Heart Association/American Stroke Association. Stroke 2010;41:2108-2129.

35. del Zoppo GJ, Ferbert A, Otis S, et al. Local intra-arterial fibrinolytic therapy in acute carotid territory stroke. A pilot study. Stroke 1988;19:307-313.

36. del Zoppo GJ, Sasahara AA. Interventional use of plasminogen activators in central nervous system diseases. Med Clin North Am 1998;82:545-568.

37. Qureshi AI, Ali Z, Suri MF, et al. Intra-arterial third-generation recombinant tissue plasminogen activator (reteplase) for acute ischemic stroke. Neurosurgery 2001;49:41-48.

38. Arnold M, Schroth G, Nedeltchev K, et al. Intra-arterial thrombolysis in 100 patients with acute stroke due to middle cerebral artery occlusion. Stroke 2002;33:1828-1833.

39. Lee DH, Jo KD, Kim HG, et al. Local intraarterial urokinase thrombolysis of acute ischemic stroke with or without intravenous abciximab: a pilot study. J Vasc Interv Radiol 2002;13:769-774.

40. Wechsler LR, Roberts R, Furlan AJ, et al. Factors influencing outcome and treatment effect in PROACT II. Stroke 2003;34:1224-1229.
41. Ogawa A, Mori E, Minematsu K, et al. Randomized trial of intraarterial infusion of urokinase within 6 hours of middle cerebral artery stroke: the Middle Cerebral Artery Embolism Local Fibrinolytic Intervention Trial (MELT) Japan. Stroke 2007;38:2633-2639.

42. Thrombolytic therapy with streptokinase in acute ischemic stroke. The Multicenter Acute Stroke Trial - Europe Study Group. N Engl J Med 1996;335:145-150.

43. Figueroa BE, Keep RF, Betz AL, Hoff JT. Plasminogen activators potentiate thrombin-induced brain injury. Stroke 1998;29:1202-1207.

44. Haley EC Jr., Thompson JL, Grotta JC, et al. Phase IIB/III trial of tenecteplase in acute ischemic stroke: results of a prematurely terminated randomized clinical trial. Stroke 2010;41:707-711.

45. Deshmukh VR, Fiorella DJ, Albuquerque FC, et al. Intra-arterial thrombolysis for acute ischemic stroke: preliminary experience with platelet glycoprotein IIb/IIIa inhibitors as adjunctive therapy. Neurosurgery 2005;56:46-54.

46. Qureshi AI, Harris-Lane P, Kirmani JF, et al. Intra-arterial reteplase and intravenous abciximab in patients with acute ischemic stroke: an open-label, dose-ranging, phase I study. Neurosurgery 2006;59:789-796.

47. Adams HP Jr., Effron MB, Torner J, et al. Emergency administration of abciximab for treatment of patients with acute ischemic stroke: results of an international phase III trial: Abciximab in Emergency Treatment of Stroke Trial (AbESTT-II). Stroke 2008;39:87-99.

48. Combined intravenous and intra-arterial recanalization for acute ischemic stroke: the Interventional Management of Stroke Study. Stroke 2004;35:904-911.

49. Hemorrhage in the Interventional Management of Stroke study. Stroke 2006;37:847-851.

50. The Interventional Management of Stroke (IMS) II Study. Stroke 2007;38:2127-2135.

51. Tomsick T, Broderick J, Carrozella J, et al. Revascularization results in the Interventional Management of Stroke II trial. AJNR Am J Neuroradiol 2008;29:582-587.

52. Nichols C, Carrozzella J, Yeatts S, Tomsick T, Broderick J, Khatri P. Is peri-procedural sedation during acute stroke therapy associated with poorer functional outcomes? J Neurointerv Surg 2010;2:67-70.

53. Mazighi M, Serfaty JM, Labreuche J, et al. Comparison of intravenous alteplase with a combined intravenous-endovascular approach in patients with stroke and confirmed arterial occlusion (RECANALISE study): a prospective cohort study. Lancet Neurol 2009;8:802-809.

54. Ribo M, varez-Sabin J, Montaner J, et al. Temporal profile of recanalization after intravenous tissue plasminogen activator: selecting patients for rescue reperfusion techniques. Stroke 2006;37:1000-1004

55. Smith WS. Safety of mechanical thrombectomy and intravenous tissue plasminogen activator in acute ischemic stroke. Results of the multi Mechanical Embolus Removal in Cerebral Ischemia (MERCI) trial, part I. AJNR Am J Neuroradiol 2006;27:1177-1182.

56. Kerber CW, Barr JD, Berger RM, Chopko BW. Snare retrieval of intracranial thrombus in patients with acute stroke. J Vasc Interv Radiol 2002;13:1269-1274.

57. Smith WS, Sung G, Starkman S, et al. Safety and efficacy of mechanical embolectomy in acute ischemic stroke: results of the MERCI trial. Stroke 2005;36:1432-1438.

58. Bose A, Henkes H, Alfke K, et al. The Penumbra System: a mechanical device for the treatment of acute stroke due to thromboembolism. AJNR Am J Neuroradiol 2008;29:1409-1413.

59. Nakano S, Iseda T, Yoneyama T, Kawano H, Wakisaka S. Direct percutaneous transluminal angioplasty for acute middle cerebral artery trunk occlusion: an alternative option to intra-arterial thrombolysis. Stroke 2002;33:2872-2876. 
60. Yoneyama T, Nakano S, Kawano H, et al. Combined direct percutaneous transluminal angioplasty and low-dose native tissue plasminogen activator therapy for acute embolic middle cerebral artery trunk occlusion. AJNR Am J Neuroradiol 2002;23:277-281.

61. Feldmann E, Daneault N, Kwan E, et al. Chinese-white differences in the distribution of occlusive cerebrovascular disease. Neurology 1990;40:1541-1545.

62. Sacco RL, Kargman DE, Gu Q, Zamanillo MC. Race-ethnicity and determinants of intracranial atherosclerotic cerebral infarction. The Northern Manhattan Stroke Study. Stroke 1995;26:14-20.

63. Nogueira RG, Schwamm LH, Buonanno FS, et al. Low-pressure balloon angioplasty with adjuvant pharmacological therapy in patients with acute ischemic stroke caused by intracranial arterial occlusions. Neuroradiology 2008;50:331-340.

64. Connors JJ III, Wojak JC. Percutaneous transluminal angioplasty for intracranial atherosclerotic lesions: evolution of technique and short-term results. J Neurosurg 1999;91:415-423.

65. Ueda T, Sakaki S, Nochide I, Kumon Y, Kohno K, Ohta S. Angioplasty after intra-arterial thrombolysis for acute occlusion of intracranial arteries. Stroke 1998;29:2568-2574

66. Suh DC, Sung KB, Cho YS, et al. Transluminal angioplasty for middle cerebral artery stenosis in patients with acute ischemic stroke. AJNR Am J Neuroradiol 1999;20:553-558.

67. Grines CL, Browne KF, Marco J, et al. A comparison of immediate angioplasty with thrombolytic therapy for acute myocardial infarction. The Primary Angioplasty in Myocardial Infarction Study Group. N Engl J Med 1993;328:673-679.

68. Black AJ, Namay DL, Niederman AL, et al. Tear or dissection after coronary angioplasty. Morphologic correlates of an ischemic complication. Circulation 1989;79:1035-1042.

69. Zaidat OO, Wolfe T, Hussain SI, et al. Interventional acute ischemic stroke therapy with intracranial self-expanding stent. Stroke 2008;39:2392-2395.
70. Abou-Chebl A, Vora N, Yadav JS. Safety of angioplasty and stenting without thrombolysis for the treatment of early ischemic stroke. J Neuroimaging 2009;19:139-143.

71. Levy EI, Siddiqui AH, Crumlish A, et al. First Food and Drug Administration-approved prospective trial of primary intracranial stenting for acute stroke: SARIS (stent-assisted recanalization in acute ischemic stroke). Stroke 2009;40:3552-3556.

72. Smith WS, Sung G, Saver J, et al. Mechanical thrombectomy for acute ischemic stroke: final results of the Multi MERCI trial. Stroke 2008;39:1205-1212.

73. De Silva DA, Fink JN, Christensen S, et al. Assessing reperfusion and recanalization as markers of clinical outcomes after intravenous thrombolysis in the echoplanar imaging thrombolytic evaluation trial (EPITHET). Stroke 2009;40:2872-2874.

74. Clark W, Lutsep H, Barnwell S, et al. The penumbra pivotal stroke trial: safety and effectiveness of a new generation of mechanical devices for clot removal in intracranial large vessel occlusive disease. Stroke 2009;40:2761-2768.

75. Castano C, Dorado L, Guerrero C, et al. Mechanical thrombectomy with the Solitaire $\mathrm{AB}$ device in large artery occlusions of the anterior circulation: a pilot study. Stroke 2010;41:1836-1840.

76. Castano C, Serena J, Davalos A. Use of the New Solitaire AB device for mechanical thrombectomy when Merci Clot Retriever has failed to remove the clot. A case report. Interv Neuroradiol 2009;15:209-214.

77. Park H, Hwang GJ, Jin SC, et al. A retrieval thrombectomy technique with the Solitaire stent in a large cerebral artery occlusion. Acta Neurochir (Wien) 2011; doi:10.1007/s00701-011-099-0.

78. Costalat V, Machi P, Lobotesis K, et al. Rescue, combined, and stand-alone thrombectomy in the management of large vessel occlusion stroke using the solitaire device: a prospective 50patient single-center study: timing, safety, and efficacy. Stroke 2011; doi:10.1161/SROKEAHA.110.608976. 